\title{
Weight Loss During Topiramate Treatment in a Severely Obese Adolescent with Congenital Adrenal Hyperplasia and Migraine
}

\author{
(D) Amy Seagroves ${ }^{1}$, (D) Heather M. Ross ${ }^{1}$, (D) Alaina P. Vidmar1,2, (D) Mitchell E. Geffner1,2,3, (D) William S. Kim¹, (D) Darryl Hwang2 \\ (D) Claudia Borzutzky2,4, (D) Nicole R. Fraga1, (D) Mimi S. Kim¹,2,3
}

${ }^{1}$ Children's Hospital Los Angeles, Center for Endocrinology, Diabetes and Metabolism, California, USA

2University of Southern California, Keck School of Medicine, Department of Pediatrics, California, USA

${ }^{3}$ The Saban Research Institute, California, USA

${ }^{4}$ Children's Hospital Los Angeles, Clinic of Adolescent and Young Adult Medicine, California, USA

\section{What is already known on this topic?}

Youth with classical congenital adrenal hyperplasia (CAH) exhibit earlier adiposity rebound, increased obesity and abdominal adiposity compared to unaffected youth. There is evidence that topiramate therapy is effective in appetite suppression resulting in body mass index reduction in obese adults and adolescents. Little is known about the efficacy of topiramate in treating severe obesity associated with $\mathrm{CAH}$

\section{What this study adds?}

Topiramate produced clinically meaningful and significant weight loss and reduced central adiposity in an adolescent with classical CAH and severe obesity. Topiramate was used safely without an increase in the frequency of adrenal crises or glucocorticoid requirement. Topiramate therapy may be especially effective in patients with $\mathrm{CAH}$, given their increased visceral adiposity.

\begin{abstract}
Youth with classical congenital adrenal hyperplasia (CAH) due to 21-hydroxylase deficiency exhibit an increased prevalence of obesity, early adiposity rebound, and increased abdominal adiposity compared to unaffected youth. Current obesity management in $\mathrm{CAH}$ largely focuses on lifestyle modifications. There is evidence that topiramate therapy is effective in reducing body mass index (BMI), as well as visceral adipose tissue (VAT), in unaffected adolescents with exogenous obesity. However, little is known about the efficacy of topiramate in patients with classical CAH. We report on a 17-year-old female with severe obesity and salt-wasting CAH due to 21-hydroxylase deficiency, who demonstrated reductions in BMI, as well as abdominal visceral and subcutaneous adipose tissue (SAT) while on topiramate therapy. The patient was diagnosed with classical CAH as a newborn with a 17-hydroxyprogesterone $11,000 \mathrm{ng} / \mathrm{dL}$. She had a BMI over the $95^{\text {th }}$ percentile at 3 years of age, followed by unremitting obesity. At 17 years old, she was started on topiramate to treat chronic migraines. Following three years of topiramate therapy, her BMI z-score decreased from +2.6 to +2.1 . After four years of therapy, her waist circumference decreased from 110 to $101 \mathrm{~cm}$, abdominal VAT decreased substantially by $34.2 \%$, and abdominal SAT decreased by $25.6 \%$. Topiramate therapy was associated with effective weight loss and reduced central adiposity in an adolescent with classical CAH and severe obesity, without any side effects. Further study is warranted regarding topiramate therapy in obese youth with classical CAH and increased central adiposity, who are at higher risk for significant morbidity.

Keywords: Congenital adrenal hyperplasia, topiramate, obesity, body composition, body fat percentage, adolescent, 21-hydroxylase deficiency
\end{abstract}

Address for Correspondence: Mimi S. Kim MD, Children's Hospital Los Angeles, Clinic of Pediatrics, California, USA

Phone: + 1 323-361-1358 E-mail: mskim@chla.usc.edu ORCID: orcid.org/0000-0002-6266-3569

Presented in: Endocrine Society Annual Meeting (ENDO 2019), New Orleans, LA, March 2019
Conflict of interest: M.S.K. and M.E.G. are Co-Directors of the CHLA

CAH Comprehensive Care Center. Received: 22.01 .2020 Accepted: 24.05.2021 


\section{Introduction}

Youth with classical congenital adrenal hyperplasia (CAH) due to 21-hydroxylase deficiency are at increased risk of earlier adiposity rebound, obesity, and increased abdominal adiposity compared to unaffected youth (1). These factors can lead to increased risks of cardiovascular disease, hypertension, and diabetes (2). However, current obesity management in $\mathrm{CAH}$ largely focuses on lifestyle modifications. Management guidelines for children with exogenous obesity recommend an intensive, in-clinic, multidisciplinary intervention, involving 26 contact hours over a six-month period (3). This may not be feasible in all clinical settings, and weight loss can be difficult to achieve through these modifications alone. Pediatric guidelines also suggest early, intensive intervention with lifestyle modifications, with consideration of anti-obesity pharmacotherapy after failure of lifestyle modifications (4). Youth with $\mathrm{CAH}$ and concomitant severe obesity are a high-risk cohort and require earlier consideration of anti-obesity pharmacotherapies. In addition, adolescents and young adults with classical $\mathrm{CAH}$ can have increased abdominal visceral adipose tissue (VAT) which is associated with inflammation, cardiovascular disease, and risk for metabolic syndrome (1). Thus, patients with CAH may also benefit from therapeutic options which specifically reduce VAT.

Topiramate is an Food and Drug Administration (FDA)approved drug utilized for the treatment of epilepsy and migraines in children. It is a GABA receptor modulator which reduces glutamate release by blocking voltage-gated $\mathrm{Na}$ channels. There is also evidence that topiramate therapy is effective in appetite suppression resulting in body mass index (BMI) reduction in obese adolescents and adults (5), with a side effect profile which can include paresthesias, cognitive slowing, and taste impairment (6). Little is known about the efficacy of topiramate in the treatment of severe obesity associated with chronic conditions such as CAH. However, as topiramate has been shown to reduce VAT more than placebo in randomized control trials, it may be particularly helpful in conditions such as $\mathrm{CAH}$ with fat distributions which involve increased VAT $(7,8)$.

We report on an adolescent case of severe obesity associated with classical CAH due to 21 -hydroxylase deficiency in which sustained BMI reduction and decreased central adiposity were achieved via topiramate therapy.

\section{Case Report}

Our female patient was diagnosed with classical, saltwasting $\mathrm{CAH}$ shortly after birth. She presented with virilized external genitalia at birth, and an elevated serum 17-hydroxyprogesterone of $11,000 \mathrm{ng} / \mathrm{dL}$ (normal < $78 \mathrm{ng} /$ $\mathrm{dL}$ ). She was treated with hydrocortisone, fludrocortisone, and salt supplementation. Molecular genetic assessment of CYP21A2 showed the presence of compound heterozygous mutations (G110del8nt and Q318X) consistent with 21-hydroxylase deficiency. She also had metopic craniosynostosis and developed generalized tonic-clonic seizures at 3 months old, requiring phenobarbital treatment for 4 years.

By 3 years of age, her BMI was 110 percent of the $95^{\text {th }}$ percentile, and remained $>95^{\text {th }}$ percentile thereafter. She developed chronic migraine headaches and was started on a topiramate dose of $50 \mathrm{mg}$ daily by her neurologist at 17 years old. The topiramate dose was titrated to $100 \mathrm{mg}$ daily over 2 years. At the time of topiramate initiation, lifestyle modifications had already been recommended as treatment for obesity, including routine exercise and nutrition counseling (e.g., a reduction of sugar-sweetened beverage intake and an increase in fruit and vegetable consumption) during clinical visits. She had only implemented participation in physical exercise at school and her neighborhood team soccer and these efforts did not result in any notable weight loss. She was on $15 \mathrm{mg} / \mathrm{m}^{2} /$ day of glucocorticoid treatment and had good hormonal control with 17-hydroxyprogesterone 59 $\mathrm{ng} / \mathrm{dL}$, androstenedione $91 \mathrm{ng} / \mathrm{dL}$, and testosterone $28 \mathrm{ng} /$ dL. She was not receiving other medications which would have had an impact on her weight. Her growth plates were fused. During the 4-year course of topiramate treatment, she continued her previous lifestyle interventions and did not start any intensive lifestyle modification programs, new therapies, prescriptive dietary regimens or major lifestyle changes. At the time of the 4-year assessment, her total daily dose of glucocorticoid had remained unchanged. Hormone analytes showed: 17-hydroxyprogesterone 221 $\mathrm{ng} / \mathrm{dL}$, androstenedione $84 \mathrm{ng} / \mathrm{dL}$, and testosterone $50 \mathrm{ng} /$ dL. She did not have any adrenal crises while on topiramate.

\section{Anthropometric Measures}

All measurements were obtained at clinical visits and during two research studies [protocols were approved by the Children's Hospital Los Angeles (CHLA) Institutional Review Board (CCI-09-00261, CHLA-14-00191)]. Written informed consent and assent were obtained from one of the parents and the patient, respectively. BMI was calculated, and the BMI z-score was reported up to 20 years of age. Waist circumference was measured, and waist-to-height ratio was calculated.

At initiation of topiramate treatment, the patient's BMI was $48.5 \mathrm{~kg} / \mathrm{m}^{2}$ (weight $100.2 \mathrm{~kg}$ ). Following 2.4 years of 
treatment, her BMI z-score decreased from +2.6 to +2.1 (Figure 1). She had the greatest BMI reduction on topiramate $100 \mathrm{mg}$ daily, with a nadir of $35.1 \mathrm{~kg} / \mathrm{m}^{2}$. Although she had a slight rebound increase in BMI of $12 \%$ during the third year of treatment, she experienced a total BMI reduction of $23 \%$ after 4.2 years of topiramate treatment. She also had a decrease in waist circumference $\left[110 \mathrm{~cm}\left(90-95^{\text {th }}\right.\right.$ percentile) to $101 \mathrm{~cm}\left(50-75^{\text {th }}\right.$ percentile)] and in her waist-to-height ratio $(0.76$ to 0.70$)$.

\section{Body Composition and Adiposity}

Body fat was measured using multiple modalities over time as part of a research protocol examining body composition

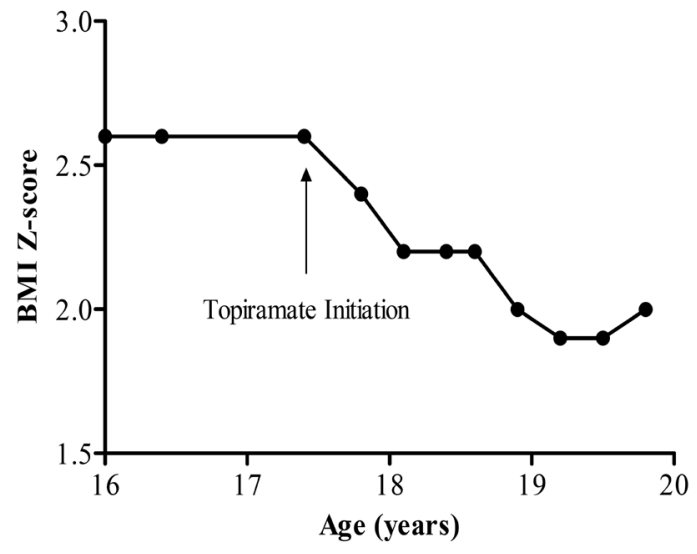

Figure 1. BMI Z-score pre- and post-topiramate initiation in a female adolescent with classical congenital adrenal hyperplasia and severe obesity. Over the course of 2.4 years from the initiation of topiramate, the BMI z-score decreased from 2.6 to 1.9 at its lowest point, suggesting that topiramate therapy resulted in a substantial and sustained weight loss over a short period of time

BMI: body mass index and adiposity distribution in patients with classical CAH. The subject had study visits at 12 and 21 years of age.

Whole body dual energy X-ray absorptiometry (DEXA; Hologic Delphi $/$ Horizon", Marlborough, MA) was utilized to examine body composition by measuring total body fat and trunk fat. Prior to topiramate treatment, the subject exhibited a high total fat mass of $48.8 \mathrm{~kg}$, a total fat percentage of $51.9 \%$, and trunk fat of $45.8 \%$. After 4 years of treatment with topiramate, she exhibited a decrease in total fat mass (48.8 to $36.7 \mathrm{~kg}$ ), total fat percent ( $51.9 \%$ to $45.9 \%$ ), and trunk fat ( $45.8 \%$ to $40 \%$ ).

In order to analyze abdominal adiposity, including VAT and subcutaneous adipose tissue (SAT), single-slice computed tomography (CT) imaging (HiLight Advantage CT, GE, Chicago, IL) was utilized at the time of the patient's initial study visit, prior to topiramate treatment. The CT abdominal axial slice corresponded to the level of the umbilicus and the L4 vertebra. The patient exhibited high amounts of abdominal adipose tissue, as can be seen in adolescents and young adults with classical CAH (9): VAT was $84.5 \mathrm{~cm}^{2}$ and SAT was $654.5 \mathrm{~cm}^{2}$. The imaging modality subsequently changed to magnetic resonance imaging (MRI) on a 3-Tesla human platform (Achieva, R5.3, Phillips Healthcare, Cleveland, $\mathrm{OH}$ ) employing a chemical-shift sequence, at the time of her next study visit four years post-initiation of topiramate therapy. For comparison across pre- and post-treatment time points, an MRI abdominal axial slice at the level of the L4 vertebra using anatomic landmarks of the musculature and orientation of the intestines to best match the initial CT single-slice taken at the second study visit was selected (Figure 2). Adipose tissue had segmented (Synapse 3D Fujifilm, Stamford, CT) on quantitative fat fraction images (9). VAT was observed to be substantially

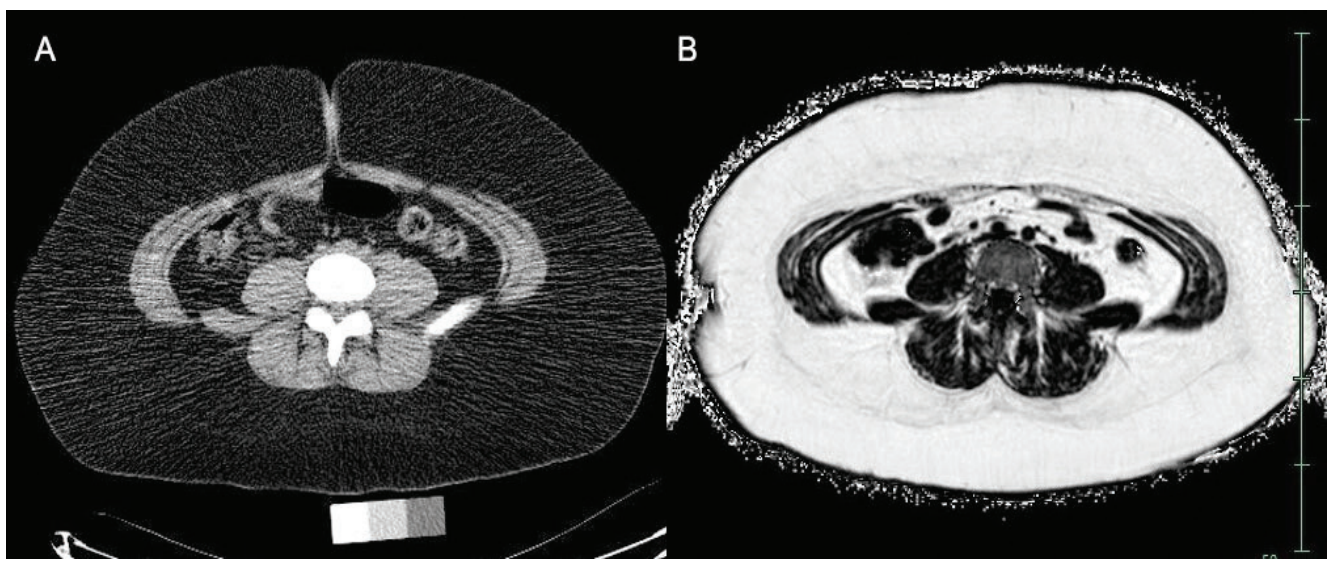

Figure 2. Abdominal adipose tissue imaging of a female adolescent with classical congenital adrenal hyperplasia to quantify visceral and subcutaneous adipose tissue. Single-slice images in the axial view, at the L4 vertebra. A) Single-slice computed tomography image was acquired at 12 years of age, prior to the initiation of topiramate treatment. B) Magnetic resonance image was acquired at 21 years of age, four years following the initiation of topiramate treatment 
decreased by $34.2 \%\left(55.6 \mathrm{~cm}^{2}\right)$ and SAT had decreased by $25.6 \%\left(486.8 \mathrm{~cm}^{2}\right)$ on the MRI. Although ideally, the comparison would have been made using images from the same modality, given the accuracy of both single-slice CT and MRI (mDixon) techniques to quantify adipose tissue, our method of comparison incorporating these modalities is supported by the current standards of imaging analysis (10).

\section{Discussion}

In our patient with classical $\mathrm{CAH}$ and severe obesity, topiramate therapy initiated for migraines produced a reasonably sustained BMI reduction over 4 years, along with reductions in total body fat and central adiposity. The BMI z-score reduction of 0.5 (23\%) which she experienced has been associated with improvements in systolic blood pressure, high-density lipoprotein cholesterol, and triglycerides in youth (11). Her dramatic reduction in VAT by over $50 \%$, along with improvements in markers of abdominal adiposity, carry additional implications for the lowering of ectopic fat accumulation, inflammation, insulin resistance, and cardiovascular disease risk $(1,12)$.

Topiramate has been demonstrated in some studies to be associated with BMI reduction in adolescents and adults with obesity which is not associated with $\mathrm{CAH}(5,13)$. However, data to support a new indication (i.e. from the U.S. FDA) for pediatric obesity, or guidelines supporting its off-label use, are lacking. Nonetheless, its clinical utilization by pediatric obesity specialists has continued to increase (14). However, more research is needed to understand topiramate standalone therapy vs. combination therapy with phentermine (15) and the off-label use of this medication.

There are several limitations to our report. Firstly, our patient was prescribed topiramate for chronic migraines, with BMI reduction being an incidental outcome. Secondly, an expanded study of topiramate use is needed in a larger number of patients with classical $\mathrm{CAH}$ and severe obesity. Importantly, topiramate did not produce adverse events in our patient, including any increase in the frequency of adrenal crises or glucocorticoid requirements, even though a report of topiramate-induced hypoadrenalism exists in an adult with $\mathrm{CAH}$ (16). Our case suggests that topiramate could be a safe pharmacological option for adolescents with $\mathrm{CAH}$ and obesity and this merits further study.

\section{Conclusion}

We conclude that topiramate treatment was associated with substantial weight loss and reduced central adiposity in an adolescent with classical $\mathrm{CAH}$ and severe obesity.
Topiramate may be especially effective in patients with $\mathrm{CAH}$ given their increased visceral adiposity. It is evident from our case and other wider applications of topiramate therapy that more research is needed in obese youth with and those without CAH. Further study is warranted on the safety and efficacy of topiramate as an adjunctive treatment in obese youth with classical $\mathrm{CAH}$ and increased central adiposity, who are at higher risk for significant morbidity.

\section{Acknowledgements}

We gratefully thank our patient and her family, the CHLA Imaging Research Program, and CHLA Clinical Trials Unit for their assistance.

\section{Ethics}

Informed Consent: Consent form was filled out by all participants.

Peer-review: Externally peer-reviewed.

\section{Authorship Contributions}

Surgical and Medical Practices: Amy Seagroves, Alaina P. Vidmar, Mitchell E. Geffner, William S. Kim, Darryl Hwang, Concept: Amy Seagroves, Darryl Hwang, Design: Amy Seagroves, Darryl Hwang, Data Collection or Processing: Amy Seagroves, Heather M. Ross, Darryl Hwang, Claudia Borzutzky, Nicole R. Fraga, Mimi S. Kim, Analysis or Interpretation: Amy Seagroves, Heather M. Ross, Alaina P. Vidmar, Mitchell E. Geffner, William S. Kim, Darryl Hwang, Claudia Borzutzky, Nicole R. Fraga, Mimi S. Kim, Literature Search: Amy Seagroves, Alaina P. Vidmar, Darryl Hwang, Writing: Amy Seagroves, Heather M. Ross, Alaina P. Vidmar, Mitchell E. Geffner, William S. Kim, Darryl Hwang, Claudia Borzutzky, Nicole R. Fraga, Mimi S. Kim.

Financial Disclosure: This work was supported by Abell Foundation (M.E.G.), CARES Foundation (CAH Comprehensive Care Center) to M.S.K. and M.E.G., and the Gustavus \& Louise Pfeiffer Research Foundation (A.S.). The writing of this paper was funded by K23 HD084735-01 A1 (NIH/NICHD) and R03 HD101718-01 (NIH/NICHD) to M.S.K. Its contents are solely the responsibility of the authors and do not necessarily represent the official views of the NIH.

\section{References}

1. Kim MS, Ryabets-Lienhard A, Dao-Tran A, Mittelman SD, Gilsanz V, Schrager SM, Geffner ME. Increased Abdominal Adiposity in Adolescents and Young Adults With Classical Congenital Adrenal Hyperplasia due to 21-Hydroxylase Deficiency. J Clin Endocrinol Metab 2015;100:1153-1159. Epub 2015 Jun 10

2. Tamhane S, Rodriguez-Gutierrez R, Iqbal AM, Prokop LJ, Bancos I, Speiser PW, Murad MH. Cardiovascular and Metabolic Outcomes 
in Congenital Adrenal Hyperplasia: A Systematic Review and MetaAnalysis. J Clin Endocrinol Metab 2019;103:4097-4103.

3. US Preventive Services Task Force; Grossman DC, Bibbins-Domingo K, Curry SJ, Barry MJ, Davidson KW, Doubeni CA, Epling JW Jr, Kemper AR, Krist AH, Kurth AE, Landefeld CS, Mangione CM, Phipps MG, Silverstein M, Simon MA, Tseng CW. Screening for Obesity in Children and Adolescents: US Preventive Services Task Force Recommendation Statement. JAMA 2017;317:2417-2426.

4. Styne DM, Arslanian SA, Connor EL, Faroogi IS, Murad MH, Silverstein $\mathrm{JH}$, Yanovski JA. Pediatric Obesity-Assessment, Treatment, and Prevention: An Endocrine Society Clinical Practice Guideline. J Clin Endocrinol Metab 2017;102:709-757.

5. Fox CK, Marlatt KL, Rudser KD, Kelly AS. Topiramate for weight reduction in adolescents with severe obesity. Clin Pediatr (Phila) 2015;54:19-24. Epub 2014 Jul 14

6. Minton GC, Miller AD, Bookstaver PB, Love BL. Topiramate: Safety and Efficacy of its Use in the Prevention and Treatment of Migraine. J Cent Nerv Syst Dis 2011;3:155-168.

7. Srivastava G, Fox CK, Kelly AS, Jastreboff AM, Browne AF, Browne NT, Pratt JSA, Bolling C, Michalsky MP, Cook S, Lenders CM, Apovian CM. Clinical Considerations Regarding the Use of Obesity Pharmacotherapy in Adolescents with Obesity. Obesity (Silver Spring) 2019;27:190-204.

8. Fox CK, Kaizer AM, Rudser KD, Nathan BM, Gross AC, Sunni M, Jennifer Abuzzahab M, Schwartz BL, Kumar S, Petryk A, Billington CJ, Ryder JR, Kelly AS. Meal replacements followed by topiramate for the treatment of adolescent severe obesity: A pilot randomized controlled trial. Obesity (Silver Spring) 2016;24:2553-2561.

9. Kim MS, Hu HH, Aggabao PC, Geffner ME, Gilsanz V. Presence of brown adipose tissue in an adolescent with severe primary hypothyroidism. J Clin Endocrinol Metab 2014;99:1686-1690. Epub 2014 Jun 10
10. Klopfenstein BJ, Kim MS, Krisky CM, Szumowski J, Rooney WD, Purnell JQ. Comparison of 3 T MRI and CT for the measurement of visceral and subcutaneous adipose tissue in humans. Br J Radiol 2012;85:826-830. Epub 2012 Apr 18

11. Reinehr T, Lass N, Toschke C, Rothermel J, Lanzinger S, Holl RW. Which Amount of BMI-SDS Reduction Is Necessary to Improve Cardiovascular Risk Factors in Overweight Children? J Clin Endocrinol Metab 2016;101:3171-3179. Epub 2016 Jun 10

12. Verrijken A, Francque S, Mertens I, Talloen M, Peiffer F, Van Gaal L. Visceral adipose tissue and inflammation correlate with elevated liver tests in a cohort of overweight and obese patients. Int J Obes (Lond) 2010;34:899-907. Epub 2010 Feb 9

13. Bray GA, Hollander P, Klein S, Kushner R, Levy B, Fitchet M, Perry BH. A 6-month randomized, placebo-controlled, dose-ranging trial of topiramate for weight loss in obesity. Obes Res 2003;11:722-733.

14. Borzutzky C, King E, Fox CK, Stratbucker W, Tucker J, Yee JK, Kumar S, Cuda S, Sweeney B, Kirk S, On Behalf Of The Power Work Group. Trends in prescribing anti-obesity pharmacotherapy for paediatric weight management: Data from the POWER Work Group. Pediatr Obes 2021;16:e12701. Epub 2020 Sep 2

15. Apovian CM, Aronne LJ, Bessesen DH, McDonnell ME, Murad MH, Pagotto U, Ryan DH, Still CD; Endocrine Society. Pharmacological management of obesity: an endocrine Society clinical practice guideline. J Clin Endocrinol Metab 2015;100:342-362. Epub 2015 Jan 15

16. Jacob K, Trainer PJ. Topiramate can induce hypoadrenalism in patients taking oral corticosteroid replacement. BMJ 2009;338:a1788. 